\title{
Synchrotron-Based X-ray Phase-Contrast Tomography for Breast Cancer Imaging
}

T.E. Gureyev 1,2,3,4,5,* , P. Baran ${ }^{1}$, S. Pacile ${ }^{6,7}$, Y.I. Nesterets ${ }^{4,5}$, S.C. Mayo ${ }^{5}$, M. Dimmock ${ }^{3}$, C. Dullin ${ }^{8}$, D. Dreossi ${ }^{6}$, F. Arfelli ${ }^{6,7}$, B. Arhatari ${ }^{9}$, D. Thompson ${ }^{4,5}$, D. Lockie ${ }^{10}$, M. McCormack ${ }^{11}$, J. Fox ${ }^{3}$, Z. Prodanovic ${ }^{3}$, S.T. Taba ${ }^{2}$, S.J. Lewis ${ }^{2}$, C. Hall ${ }^{12}$, D. Hausermann ${ }^{12}$, F. Zanconati ${ }^{13}$, M. Cholewa ${ }^{14}$, H. Quiney ${ }^{1}$, G. Tromba ${ }^{6}$, ..G. Peele $^{9,12}$, P.C. Brennan ${ }^{2}$

${ }^{1}$ The University of Melbourne, Parkville 3010, Australia

${ }^{2}$ The University of Sydney, NSW 2006, Australia

${ }^{3}$ Monash University, Clayton 3800, Australia

${ }^{4}$ University of New England, Armidale 2351, Australia

${ }^{5}$ Commonwealth Scientific and Industrial Research Organisation, Clayton 3168, Australia

${ }^{6}$ Elettra - Sincrotrone, 34149 Basovizza, Trieste, Italy

${ }^{7}$ University of Trieste, 34127 Trieste, Italy

${ }^{8}$ University Hospital Goettingen, 37075 Goettingen, Germany

${ }^{9}$ La Trobe University, Bundoora 3086, Australia

${ }^{10}$ Maroondah BreastScreen, Ringwood East 3135, Australia

${ }^{11}$ Dorevitch Pathology, Heidelberg 3084, Australia

${ }^{12}$ Australian Synchrotron, ANSTO, Clayton 3168, Australia

${ }^{13}$ Academic Hospital of Trieste, 34128 Trieste, Italy

${ }^{14}$ University of Rzeszow, 35-310 Rzeszow, Poland

* Corresponding author, timur.gureyev@unimelb.edu.au

Breast cancer is one of the two leading causes of cancer fatalities for women in all countries [1]. Early detection can be crucial for successful treatment of this often aggressive type of cancer, before it spreads to vital organs. Regular mammographic screening is therefore recommended by health authorities in many countries as an optimal strategy for detecting the first signs of breast cancer [2]. Concerns about harm from false positive and false negative diagnoses [2] create a considerable incentive for development of improved mammographic techniques. Improving sensitivity and specificity of existing breast cancer imaging technologies, could improve detection [3]; doing this while delivering reduced Xray dose to the patient, and, preferably, also removing or reducing the considerable physical discomfort experienced by many patients during the conventional breast screening procedures [4-9] would be an added benefit. Propagation-based phase-contrast X-ray CT (PB-CT), which exploits refraction (in addition to absorption) of X-rays in tissue, shows particular promise in this regard due to its superior sensitivity to soft-tissue tumors [5-17]. The improvement in the image quality due to the use of phase contrast, combined with the reduced radiation dose and less physical discomfort associated with the procedure, has the potential to significantly increase the participation rate of women in regular breast screening. This in turn will ultimately decrease the mortality and morbidity associated with breast cancer through improved early detection.

Currently, PB-CT breast imaging is feasible only at suitable synchrotron beamlines; the low brightness of microfocus laboratory X-ray sources that have sufficient spatial coherence for PB-CT phase contrast imaging makes exposure times impractically long or signal to noise (SNR) unacceptably poor. Demonstrating definitive benefits through breast cancer imaging facilities at synchrotrons provides a strong motivation for developing systems suitable for use in hospitals. In our recent experiments at IMBL at the Australian Synchrotron and at SYRMEP beamline at Elettra synchrotron in Trieste [10-17], 
we have analysed the essential parameters of PB-CT technique relevant to breast cancer imaging, including the optimal X-ray energy range, sample-to-detector distances and key detector characteristics. We have theoretically proved $[12,18]$ and confirmed,in preliminary experiments, that synchrotron PBCT increases the SNR in breast cancer imaging by a factor of 10 or more at the same radiation dose. Equivalently a decrease in the radiation dose by a factor of 100 or more at the same SNR, compared to conventional absorption-based CT, can be obtained with minimal loss of spatial resolution. Such a result provides a robust justification for the use of PB-CT breast cancer imaging of live patients at synchrotron facilities similar to IMBL and SYRMEP in order to demonstrate clinical effectiveness. Continuing work is aimed at addressing some remaining challenges on the way to practical implementation of medical breast imaging with synchrotron radiation. In particular, we need to prove that the sensitivity and specificity of PB-CT in breast cancer imaging are higher than in other breast imaging techniques available at present.
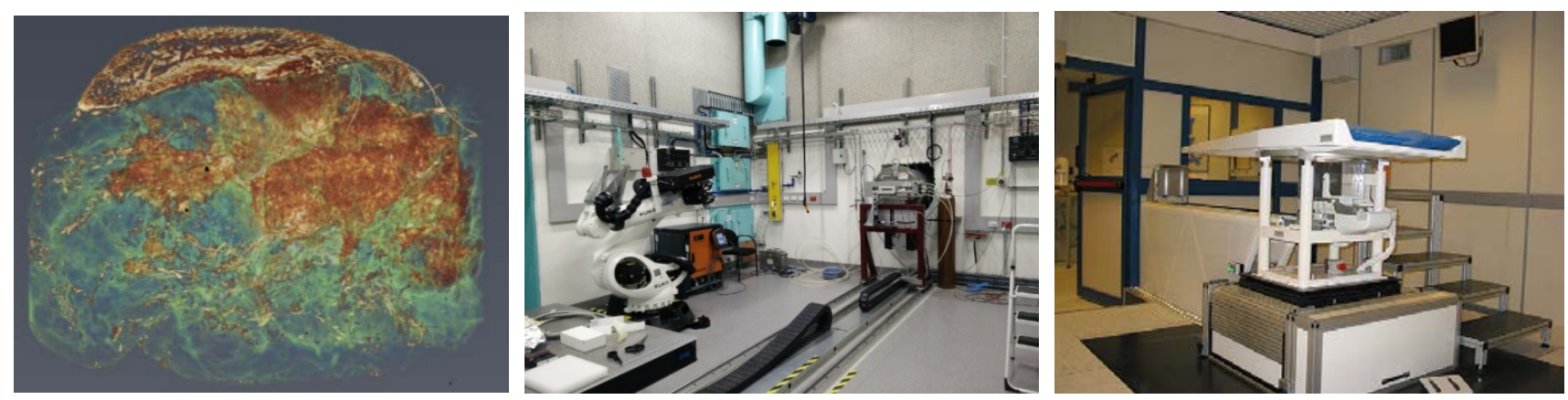

Fig. 1. (a) CT reconstruction of a fresh mastectomy sample with cancer lesions; (b) Hutch 3B of the IMBL, Australian Synchrotron, where PB-CT breast cancer imaging will be implemented; (c) Patient support table for breast CT imaging at SYRMEP beamline at Elettra Synchrotron (Trieste, Italy).

[1] LA Torre et al CA Cancer J Clin 65 (2015) 87.

[2] M Løberg, Breast Cancer Res. 17 (2015) 63.

[3] ED Pisano, M.J. Yaffe, JAMA 311 (2014) 2488.

[4] A Malliori et al., Med.Phys. 39 (2012) 5621.

[5] Y Zhao et al., Proc.Nat.Acad.Sci. 109 (2012) 18290.

[6] PC Diemoz et al., Opt. Express 20, (2012) 27670.

[7] A Bravin et al., Phys. Med. Biol. 58 (2013) R1.

[8] A Olivo et al., Phil.Trans,Roy.Soc. A (2014) 1.

[9] E Brun et al., Med.Phys. 41 (2014) 11192.

[10] TE Gureyev et al., J. Phys. D: Appl. Phys. 47 (2014) 365401.

[11] TE Gureyev et al., J.Appl.Phys. 114 (2013) 144906.

[12] YI Nesterets et al., J.Phys.D :Appl.Phys. 47 (2014) 105402.

[13] TE Gureyev et al., Opt.Express 22 (2014) 9087.

[14] YI Nesterets et al., J.Synch.Rad., 22 (2015) 1509.

[15] S Pacile et al., Biomed.Opt.Express, 6 (2015) 3099.

[16] G Tromba et al., Lect.NotesComput.Sci. 9699 (2016) 625.

[17] PM Baran et al., Phys.Med.Biol. 62 (2017) 2315.

[18] TE Gureyev et al, J.Opt.Soc.Am. A 34 (2017) 2251.

[19] The authors acknowledge funding from Project Grant 1138283 “Towards the clinical application of phase-contrast computed tomography in breast cancer imaging”, NHMRC, Australia. 